\title{
Enhanced Load Frequency Control: Incorporating Locational Information for Temporal Enhancement
}

ISSN 1751-8644 doi: 0000000000 www.ietdl.org \author{
Prostejovsky ${ }^{2}$, and Evangelos Rikos ${ }^{3}$ \\ ${ }^{1}$ Institute for Energy and Environment, University of Strathlcyde, Glasgow, G1 1RD, UK \\ ${ }^{2}$ Department of Electrical Engineering, Technical University of Denmark, Riso, 4000, Denmark \\ ${ }^{3}$ Center for Renewable Energy Sources and Saving, Pikermi Attiki, 19009, Greece \\ ${ }^{*}$ E-mail: mazheruddin.syed@strath.ac.uk
}

Mazheruddin H. Syed ${ }^{1}$, Efren Guillo-Sansano ${ }^{1}$, Steven M. Blair ${ }^{1}$, Graeme M. Burt ${ }^{1}$, Alexander M.

\begin{abstract}
With the increasing penetration of renewables in power systems, frequency regulation is proving to be a major challenge for system operators using slower conventional generation units, and alternative means to provide faster regulation are being actively sought. The participation of demand side management in ancillary service provision is proven in a number of energy markets, yet its full potential to benefit frequency regulation, including the exploitation of fast power ramping capability of some devices, is still undergoing research. In this paper, a novel approach to improve the speed of response of load frequency control, a secondary frequency control, approach is proposed. The proposed control is enabled by an effective location identification technique, is highly resilient to anticipated system changes such as reduction of inertia, and enables fully decentralized power system architectures. The effectiveness of the approach is demonstrated and compared to that of present day regulation control, by means of real-time simulations incorporating appropriate time delays conducted on a five-area reduced model of the Great Britain power system. The applicability of the method is further proven under realistic communications delays and measurements experimentally using a controller and power hardware-in-the-loop setup, demonstrating its critical support for enabling the stable operation of future power systems.
\end{abstract}

\begin{tabular}{ll} 
Nomenclature \\
\multicolumn{1}{l}{ Textual Abbreviations } \\
ACE & area control error \\
ADD & area disturbance determiner \\
BCL & balance control loop \\
BESS & battery energy storage system \\
CG & conventional generation \\
CLFC & conventional load frequency control \\
DSA & demand side aggregator \\
ELFC & enhanced load frequency control \\
FCDM & frequency control by demand management \\
GB & Great Britain \\
LFC & load frequency control \\
PFC & primary frequency control \\
PI & proportional integral \\
RoCoF & rate of change of frequency \\
SFC & secondary frequency control \\
Mathematical Formulations \\
$\alpha D S A i, \alpha C G i$ & participation factors for demand side aggregators \\
& and conventional generators respectively \\
$\beta_{i}$ & frequency bias factor for area $i$ \\
$\delta_{i j}$ & area $i$ and $j$ tie-line breaker state \\
$\Delta f_{i}$ & change in frequency of area $i$ \\
$\Delta f^{o v r}$ & relative frequency overshoot \\
$\Delta P$ & power imbalance \\
$\Delta P_{i}^{B C L}$ & control effort of auxiliary controller \\
$\Delta P_{c i}$ & control effort of secondary control \\
$\Delta P_{i}^{D S A^{*}}$ & demand side aggregator power regulation \\
$\Delta P_{g i}$ & deviation in generator valve position \\
$\Delta P_{L i}$ & load power variation for area $i$ \\
$\Delta P_{m i}$ & deviation in generator mechanical output \\
$\Delta P_{i}^{t i e}$ & tie-line power deviation for area $i$ \\
$\Delta P_{T h, i}^{t i e}$ & tie-line power deviation threshold \\
&
\end{tabular}

\begin{tabular}{|c|c|}
\hline$\Delta P_{i}^{t i e, *}$ & $\begin{array}{l}\text { filtered tie-line power deviation } \\
\text { error margin }\end{array}$ \\
\hline$\mu_{\text {step }}, \mu_{\text {inertia }}$ & step size and inertia multipliers respectively \\
\hline$\mu, \sigma$ & mean value and standard deviation respectively \\
\hline$\Sigma \Delta P_{i}^{D S A}$ & demand side aggregator power variation for area $i$ \\
\hline & synchronous area \\
\hline$\frac{d f}{d t}, \frac{d f}{d t}^{*}, \frac{d f}{d t}^{* *}$ & $\begin{array}{l}\text { rate of change of frequency: measured, extremum, } \\
\text { and filtered respectively }\end{array}$ \\
\hline$d_{i}$ & output of area disturbance determiner \\
\hline$D_{i}$ & load damping coefficient for area $i$ \\
\hline$f_{d b}$ & frequency dead-band \\
\hline$f_{\text {nom }}, f_{\text {meas }}$ & nominal and measured frequency respectively \\
\hline$f_{\max }, f_{\min }$ & maximum and minimum frequency respectively \\
\hline$H_{i}, H_{0}$ & $\begin{array}{l}\text { inertia constant for area } i \text { and reference inertia of } \\
\text { the system respectively }\end{array}$ \\
\hline$i$ & index representing area under consideration \\
\hline $\begin{array}{l}j \\
k\end{array}$ & $\begin{array}{l}\text { index representing adjacent areas to area indexed } i \\
\text { scaling factor }\end{array}$ \\
\hline$K_{P i}, T_{I i}$ & $\begin{array}{l}\text { proportional gain and integral time constant of } \\
\text { secondary controller for area } i \text { respectively }\end{array}$ \\
\hline$K_{P, a u x}, T_{I, a u x}$ & $\begin{array}{l}\text { proportional gain and integral time constant of } \\
\text { auxiliary controller for area } i \text { respectively }\end{array}$ \\
\hline$M$ & number of control areas \\
\hline $\mathcal{N} \mathcal{H}_{i}$ & neighborhood of area \\
\hline$P^{s t e p}, P_{0}^{\text {step }}$ & disturbance size and reference disturbance size \\
\hline $\begin{array}{l}P_{0, i}^{\text {tie }}, P_{\text {meas }, i}^{\text {tie }} \\
R_{i}\end{array}$ & $\begin{array}{l}\text { scheduled and measured tie-line power flow } \\
\text { speed droop for area } i\end{array}$ \\
\hline$T_{a i}, T_{b i}$ & turbine time constants for area $i$ respectively \\
\hline$T_{g i}$ & governor time constant for area $i$ \\
\hline$t_{h}, t_{k}$ & control time step and a chosen time instant \\
\hline$T_{f}$ & filter time constant \\
\hline & measurement window length \\
\hline & frequency restoration time \\
\hline$u, w, x$ & control, disturbance and state vectors respectively \\
\hline
\end{tabular}




\section{Introduction}

Increasing penetration of renewable generation sources, the decreasing population of conventional synchronous generation (CGs) and the consequent decrease in system inertia deteriorates the system frequency response. In a study undertaken by the Australian Energy Market Operator, it was shown that with the forecasted change in generation mix (i.e., a rapid uptake of non-synchronous generation), the rate of change of frequency (RoCoF) levels will increase such that by 2022 there would be less than two seconds for primary frequency control (PFC) actions to arrest frequency before it declines below the defined containment band [1]. The two seconds period is much shorter than the response time typically observed for CGs. It can therefore be said that the decreasing inertia in the network imposes new requirements on existing services. In recent literature, a number of works have proposed novel synthetic-inertial control approaches [2-5] to alleviate the problem of declining system inertia; however, in practice synthetic inertia is commercially unavailable [6]. Furthermore, as reported in [6], virtual inertia emulation is feasible within power networks with high inertia, but it becomes increasingly challenging to obtain an appropriate transient response in low inertia circumstances. Owing to the adaptable/flexible nature of power electronic interfaced devices, they can yield a wider range of responses, and enforcing only inertial response from such devices can be limiting and can curb further developments. Other system operators around the world have noted this common concern of declining system inertia and its effect on system frequency and the challenges brought forwards towards frequency control [7, 8]. Realising the need for faster acting service provision, system operators are working towards better understanding of the impact and integration of fast response in the entire spectrum of frequency control mechanisms (such as inertial, PFC, secondary frequency control), as is evident from the pilots and plans of many international system operators summarized in [9].

Load frequency control (LFC), also referred to as automatic generation control, is a secondary frequency control (SFC) process employed to maintain the frequency of interconnected powersystems such as in Continental Europe, USA and Australia [10-12]. LFC is relatively slow, designed for responding only after the frequency has been contained by the PFC. The performance of LFC can conventionally be improved by the use of fast acting gas turbines as the regulation providing units; however, their availability and cost of operation can restrict their implementation [13]. An alternative to the conventional approach is the use of flexible demand available within the network. In [14] and [15], it has been shown that the utilization of a utility scale battery energy storage system (BESS) in conjunction with CGs improves the LFC delivering performance of the CGs. In a study that characterizes the effectiveness of utilizing battery energy storage systems for LFC compared to CGs, a reduction in the regulation requirement is observed even when they constitute a small portion of total capacity [16]. This has also been confirmed in studies conducted by [17]. Although the performance of LFC is improved by utilizing fast acting devices, the slow operation design of LFC, due to ramp rate constraints on CGs, does not allow for exploitation of the full potential of such devices.

An approach, already in use in the Great Britain (GB) power system and referred to as frequency control by demand management (FCDM) [18], provides an alternative means to exploit the power ramping potential of fast acting devices. This approach is based on determining a frequency threshold for participating fast acting devices [19]. The amount of reserves activated by such means is subtracted from the LFC regulation command. In [20], it was shown that such an approach alleviates the burden on LFC and provides a better frequency response. At present, only relatively large commercial customers participate in FCDM and are activated by means of under-frequency relays. The dimensioning of FCDM reserves and the thresholds for their activation are carefully determined based on the system power frequency characteristics. In the future, with higher penetration of intermittent renewable energy resources within the grid, this reserve dimensioning and thresholds determination will prove to be even more challenging as highly variable system power frequency characteristics are anticipated. The opportunity of participating in FCDM will be extended to the large amount of flexibility foreseen to be available within the grid (including domestic devices such as electric vehicles and heat pumps). Devices interfaced by power electronics will have no difficulty in participating through local measurements, however, some devices that inherently do not incorporate local observables may not be able to participate. In other words, the large amount of distributed domestic flexibility within the network will be available to participate through the traditional LFC (as it requires communications enablement rather than local measurements).

In [21-23], alternative structures for LFC and controls for the participation of fast acting devices have been proposed. The participation of demand side fast acting devices in LFC structures has further been facilitated by the establishment of demand side aggregators (DSA) that can be perceived as any other regulating reserve-providing unit under LFC [24].

To summarize, it can be said that in the past there had been no incentive to improve the speed of response of SFC. With the changes expected within the power system in near future, there is a growing interest in improving the speed of response of frequency control services, including SFC. Improving the response speed of SFC: (i) is limited due to the ramp rate constraints on participating CGs, and (ii) can deteriorate the system frequency response due to SFC interaction with the PFC response. As discussed above, even with the increasing presence of fast acting devices within the network, the speed of response of SFC within the conventional architecture of LFC cannot be improved.

To address the above gaps, in this paper a novel SFC is proposed. The proposed control incorporates locational information, i.e., the location of the disturbance (power imbalance due to loss/addition of generation or load is considered a disturbance in this paper), to enable the temporal enhancement of LFC. The proposed control comprises a fast acting balance control loop (BCL), in addition to the secondary control loop of LFC. The BCL comprises an area disturbance determiner, capable of fast, autonomous and definitive identification of disturbance location. The fast identification of the disturbance location allows for unilateral activation of reserves only within the area where the disturbance has occurred at increased speeds which mitigates the deteriorating impact of its interaction with PFC. In addition, the control effort to fast acting resources is a combination of effort from BCL and secondary control loop, while the control effort to slower acting resources is from secondary control loop only, thereby eliminating the restrictions on ramp rates conventionally imposed. The proposed control is simple and does not require additional forecasting or complete network awareness, thereby allowing seamless integration within present day LFC. The performance of the proposed control is compared with that of conventional LFC within a reduced five-area dynamic model of the Great Britain power system by real-time simulations, corroborated analytically by small-signal analysis and its real world applicability proven by experimental evaluation within a laboratory.

\section{Proposed Frequency Control Approach}

In this section, to present the proposed approach effectively, the conventional LFC (referred to as CLFC henceforth) is first introduced and its shortcomings highlighted. This is followed by the description of the proposed control approach.

\subsection{Conventional Load Frequency Control (CLFC)}

Consider an interconnected power system with $M$ control areas indexed by $i=1,2, \cdots, M$. The CLFC model of the $i^{t h}$ control area is presented in Fig. 1. As shown, the CLFC comprises a primary control loop and a secondary control loop, where the aim of primary control is to contain the frequency deviation caused by power imbalance in any control area, while the secondary control is responsible to recover the frequency back to its nominal value [5]. The system dynamics of the $i^{t h}$ area can be represented by the following 


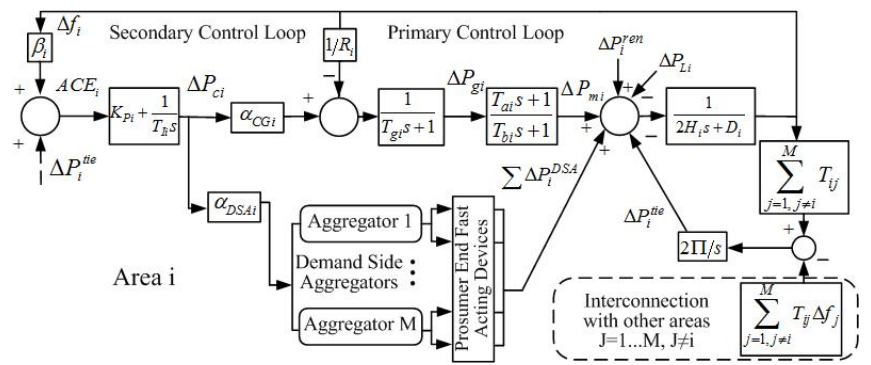

Fig. 1: Conventional load frequency control (CLFC)

differential equations:

$$
\begin{array}{r}
\Delta \dot{f}_{i}(t)=-\frac{D}{2 H_{i}} \Delta f_{i}(t)+\frac{1}{2 H_{i}}\left(\Delta P_{m i}(t)+\Delta P_{i}^{r e n}(t)\right. \\
\left.-\Delta P_{L i}(t)-\Delta P_{i}^{t i e}(t)+\Sigma \Delta P_{i}^{D S A}(t)\right)
\end{array}
$$

$\Delta \dot{P}_{m i}(t)=-\frac{1}{T_{b i}} \Delta P_{m i}(t)+\frac{1}{T_{b i}} \Delta P_{g i(t)}+\frac{T_{a i}}{T_{b i}} \Delta \dot{P_{g i}}(t)$

$\Delta \dot{P_{g i}}(t)=-\frac{1}{T_{g i}} \Delta P_{g i}(t)+\frac{1}{T_{g i}} \alpha_{C G i} \Delta P_{c i}(t)-\frac{1}{R_{i} T_{g i}} \Delta f_{i}(t)$

where

$$
\Delta P_{i}^{t i e}=\frac{2 \pi}{s}\left[\sum_{j=1, j \neq i}^{M} T_{i j}\left(\Delta f_{i}-\Delta f_{j}\right)\right]
$$

where $\Delta f_{i}$ is the change in frequency, $\Delta P_{c i}$ denotes control effort of secondary control, $\Delta P_{m i}$ and $\Delta P_{g i}$ are the deviations of generator mechanical output and valve position for area $i$ respectively. $H_{i}, D_{i}, R_{i}, T_{g i}, T_{a i}$ and $T_{b i}$ are the inertia constant, load damping coefficient, speed droop, governor and turbine time constants for area $i$ respectively. $\Delta P_{i}^{r e n}, \Delta P_{L i}, \Delta P_{i}^{t i e}$ and $\Delta P_{i}^{D S A}$ are power variations of renewable generation, loads, tie-line and DSAs, respectively, which can be viewed as external disturbances to the system. $\Delta f_{j}$ is the change in frequency of area $j$ and $T_{i j}$ is the synchronizing torque. The secondary control input is a signal referred to as the area control error (ACE) and can be defined as

$$
A C E_{i}(t)=\beta_{i} \Delta f_{i}(t)+\Delta P_{i}^{t i e}(t)
$$

where $\beta_{i}$ is the frequency bias factor. Upon occurrence of an event that leads to a deviation in frequency and ACE beyond a set threshold, a proportional integral (PI) control is employed to force the ACE to zero and can be represented as

$$
\Delta P_{c i}(t)=-K_{P i} A C E_{i}(t)-\frac{1}{T_{I i}} \int A C E_{i}(t)
$$

where $K_{P i}, T_{I i}$ are the proportional gain and integral time constant, respectively.

\subsection{Shortcomings of Conventional Load Frequency Control}

Theoretically, the distinction between primary and secondary frequency control is their control objective, i.e., primary contains frequency deviations and secondary restores the frequency to its nominal value. Practically, a time scale of operation is also associated with the two controls, i.e. the primary response is expected within ten seconds of the observed disturbance while secondary response can vary from thirty seconds to minutes. This is, in general, regarded as the secondary control intentionally being designed

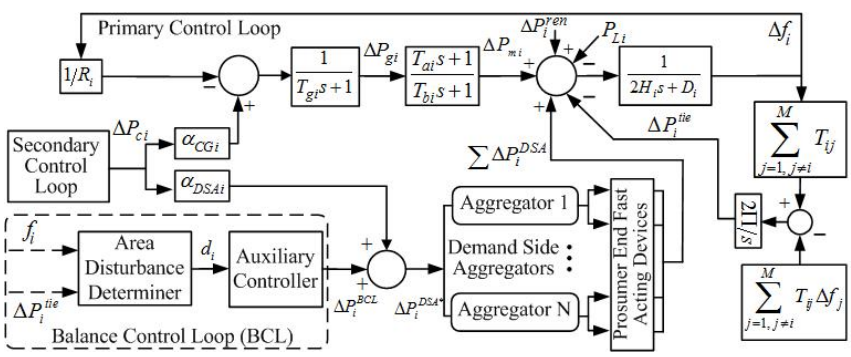

Fig. 2: Enhanced load frequency control (ELFC)

for much slower operation than primary control. However, this association of time with control objective is due to practical limitations as identified below:

1. Slow disturbance location identification: Within CLFC, although the entire synchronous area perceives the disturbance as a change in frequency, only the area with the disturbance is responsible to restore the frequency. This decentralized operation is achieved by means of ACE, i.e., assuming appropriate calculation of $\beta$, the ACE distinguishes an internal disturbance (within the area) from an external one (outside the area). A definitive identification can only be obtained when the RoCoF $\frac{d f}{d t}=0$, and hence the delay in the operation of the secondary control.

2. Transient ACE following a disturbance: Following a disturbance, the ACE of non-disturbance areas slowly converges to zero. If the speed of response of secondary control was increased, the transient non-zero ACE would enforce unnecessary secondary participation from non-disturbance areas. This can further be exacerbated when $\beta$ is miscalculated.

3. Ramp-rate constraint of CGs: Large CGs constitute the majority of the secondary reserves. The ramp rate constraint of these participating generators limits the speed of response of secondary control.

\subsection{Proposed Enhanced Load Frequency Control (ELFC)}

To overcome the limitations of the conventional approach, a novel enhanced LFC (ELFC), as illustrated in Fig. 2, is proposed. The control incorporates a fast acting $\mathrm{BCL}$, in addition to the primary and secondary control loops. The key features of the proposed approach are as follows:

- The BCL comprises an area disturbance determiner (ADD), capable of fast and autonomous identification of disturbance location in a completely decentralized manner. This ensures activation of reserves unilaterally only within the area that has initiated the disturbance, allowing for faster response speeds (faster reserve activations).

- The area power imbalance observed over tie-lines $\left(\Delta P_{i}^{t i e}\right)$ is the control input of BCL as opposed to the conventionally utilized ACE. The non dependence of control input on frequency eliminates deteriorating interaction with primary control and avoids unnecessary activations.

- The BCL employs only fast acting demand side devices through DSAs as regulation providing reserves, eliminating any restriction on speed of response due to ramp-rate constraints. Therefore, the power regulation command of the DSAs comprises two parts: (i) regulation command from secondary control loop with predefined participation factor $\alpha_{D S A i}$ and (ii) regulation command from the BCL.

The fast acting nature of the BCL enables active contribution to objectives of both, primary and secondary control, hence justifying its existence as an independent loop. The description of the fast acting $\mathrm{BCL}$ is presented in the following section. 


\section{Fast Acting Balance Control Loop (BCL)}

In this section, the operation of the fast acting BCL is explained.

\subsection{Fast Location Identification by Area Disturbance Determiner (ADD)}

The objective of the ADD is to autonomously, quickly and unambiguously distinguish between disturbances originating within the area and those originating elsewhere in the synchronous region. The operating principle of the ADD is presented below.

Any sudden imbalance between generator input mechanical power and load will lead to a deviation in system frequency, the dynamics of which can be represented by the swing equation as

$$
\Delta P=\frac{2 H}{f_{n o m}} \frac{d f}{d t}
$$

where $\Delta P$ is the power imbalance in the system, $H$ is the inertia constant of the system, $f_{n o m}$ is the nominal frequency and $\frac{d f}{d t}$ is the RoCoF. RoCoF at a specific time instant $t_{k}$ can be estimated as

$$
\frac{d f}{d t}=\frac{f_{t_{k}}-f_{t_{k}, T_{n}}}{T_{n}}
$$

where $T_{n}$ is the length of the measuring window. Within a synchronous area $\mathcal{A}$ and for area under consideration $i$, the neighborhood can be defined as $\mathcal{N} \mathcal{H}_{i}=\left\{i, \mathcal{A N} \mathcal{N}_{i}\right\} \subseteq \mathcal{A}$. With $j \in \mathcal{A N}_{i}$ as adjacent areas coupled over tie-lines with breaker state $\delta_{i j}$, the change in tie-line power flow for area $i$, subject to the disturbance, can be calculated as

$$
\Delta P_{i}^{t i e}=\sum_{j \in \mathcal{A N}_{i}} \delta_{i j} P_{0, i}^{t i e}-\sum_{j \in \mathcal{A N}_{i}} \delta_{i j} P_{\text {meas }, i}^{\text {tie }}
$$

where $P_{0, i}^{t i e}$ and $P_{\text {meas }, i}^{\text {tie }}$ are the scheduled and measured tie-line power flows respectively. As the change in electric power flow is immediate, upon occurrence of power imbalance such that $\Delta P_{i}^{t i e} \geq$ $\Delta P_{T h, i}^{t i e}$, measuring the extremum of $\frac{d f}{d t}$ as $\frac{d f}{d t}{ }^{*}$, for $\Delta P_{i}^{t i e}, \frac{d f}{d t}{ }^{*} \neq$ 0 , the disturbance is classified as originating within the area if

$$
\begin{aligned}
& \left\lfloor\frac{\frac{d f}{d t}}{\left|\frac{d f}{d t}\right|+1}\right\rfloor-\left\lfloor\frac{-\frac{d f}{d t}{ }^{*}}{\left|\frac{d f}{d t}\right|+1}\right\rfloor \\
& \neq\left\lfloor\frac{\Delta P_{i}^{t i e}}{\left|\Delta P_{i}^{t i e}\right|+1}\right\rfloor-\left\lfloor\frac{-\Delta P_{i}^{t i e}}{\left|\Delta P_{i}^{t i e}\right|+1}\right\rfloor
\end{aligned}
$$

where $\Delta P_{T h, i}^{t i e}$ is the threshold of change in tie-line power flow set based on the largest expected load variation in area $i$. It should be noted that Eq. (10) is an alternative form of signum function using floor $(\lfloor\rfloor)$ and absolute $(||)$ functions. Such representation is more appropriate in this case due to its use for evaluation of real numbers except zero, i.e., for $\Delta P_{i}^{t i e}, \frac{d f}{d t} * 0$. An alternative representation for convenience of implementation in embedded platforms is with the use of Iversons brackets [25] as $-\left[\frac{d f}{d t}^{*}<0\right]+\left[\frac{d f}{d t} * 0\right] \neq$ $-\left[\Delta P_{i}^{t i e}<0\right]+\left[\Delta P_{i}^{t i e}>0\right]$. Upon satisfying Eq. (10), i.e., the sign of $\Delta P_{i}^{t i e}$ is not equal to the sign of $\frac{d f}{d t}{ }^{*}$, the output of the disturbance determiner is $d_{i}=\Delta P_{i}^{t i e}$ indicating the disturbance to be within the area, else $d_{i}=0$ indicating the disturbance is outwith the area. Therefore, each area of $\mathcal{A}$ can identify disturbances originating within themselves based on local measurements in a decentralized manner. Although the identification is fast, there might be a frequency dead-band, $\left(f_{d b}\right)$ such that for measured frequency $f_{\text {meas }}$, no frequency control measure is employed when $-f_{d b}<$ $f_{\text {meas }}-f_{\text {nom }}<f_{d b}$

\subsection{Auxiliary Controller}

The output of ADD is an input to the auxiliary controller, and for when Eq. (10) is true, $d_{i}$ should be controlled to zero. Employing a PI control, the control effort can be represented as

$$
\Delta P_{i}^{B C L}(t)=-K_{P, a u x} d i(t)-\frac{1}{T_{I, a u x}} \int d_{i}(t)
$$

where $K_{p, a u x}, T_{I, a u x}$ are the proportional gain and integral time constant of the auxiliary controller respectively. Contrary to the secondary control loop, where the value of $T_{I}$ is limited by the ramp rate constraints of CGs, $T_{I, \text { aux }}$ can be chosen to achieve a faster response. The use of PI control for BCL is not mandatory and the output of ADD can be an input for direct load frequency control as presented in [26]. The effective power regulation command issued to the DSAs can be represented as

$$
\Delta P_{i}^{D S A^{*}}(t)=\Delta P_{i}^{B C L}(t)+\alpha_{D S A i} \Delta P_{c i}(t)
$$

\subsection{Low Pass Filtering}

To suppress the measurement noise and to smoothen the response of BCL, a low pass filter is utilized for $\Delta P_{i}^{t i e}$ and $\frac{d f}{d t}$. The filtered signals at time instant $t_{k}$ can be represented as

$$
\begin{gathered}
\Delta P_{i}^{t i e, *}\left(t_{k}\right)=(1-a) \Delta P_{i}^{t i e, *}\left(t_{k}-1\right)+a \Delta P_{i}^{t i e}\left(t_{k}\right) \\
\frac{d f}{d t}^{* *}\left(t_{k}\right)=(1-a) \frac{d f}{d t}^{* *}\left(t_{k}-1\right)+a \frac{d f}{d t}\left(t_{k}\right)
\end{gathered}
$$

where $a=\frac{t_{h}}{T_{f}+t_{h}}$, with $t_{h}$ as the time step of control implementation and $T_{f}$ as the filter time constant. It is important to choose the filter time constant such that it renders satisfactory quality of input while attributing least delay.

\section{Test System Characterization}

In this paper, the CLFC is the reference implementation against which the performance of the proposed ELFC will be assessed. In the following sub-sections, the test system characteristics are detailed.

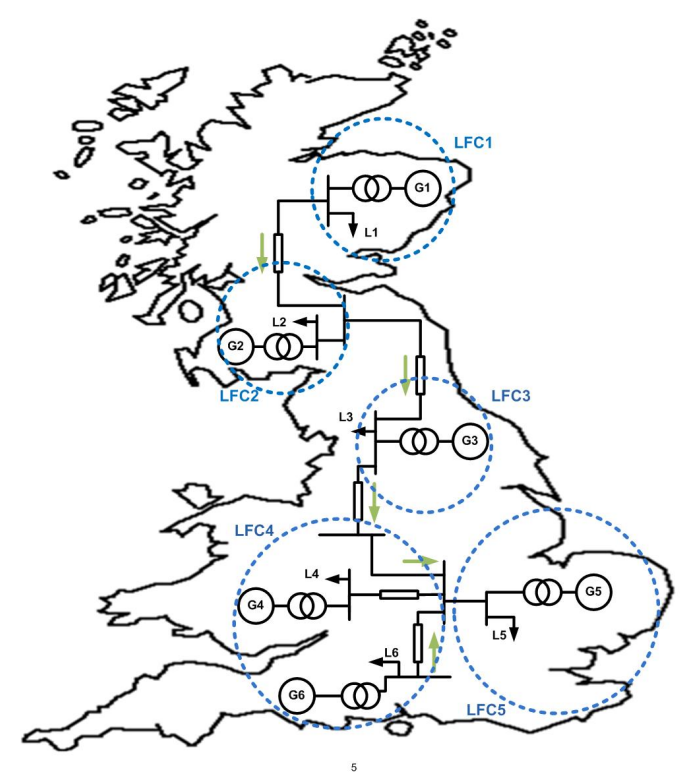

Fig. 3: Reference five-area reduced GB power system. 
$\alpha_{D S A i}=0.75$, while $f_{d b}$ is selected as $0.1 \mathrm{~Hz}$ [28], [33], [11].

For analyzing the proposed frequency control approach in this paper, a reduced six-machine dynamic model of the GB power system has been chosen as the test grid. It should be noted that the GB grid operates as one synchronous area. However, in this paper, the buses of the reduced model have been grouped to represent multiple LFC areas as shown in Fig. 3, each of which relates to one or more regions identified in the GB National Electricity Transmission System boundary map presented in the Electricity Ten Year Statement of the Transmission System Operator [27].

4.1.1 Modeling: The model has been developed in RSCAD and simulated in real-time using a digital real-time simulator from RTDS Technologies, with each area comprising at least one aggregated generator and an aggregated load. Each aggregated generator is modeled as a large synchronous machine connected to the transmission system via a step-up transformer $(13.8 / 400 \mathrm{kV})$. The rating of each generator is set according to a characteristic GB 2017 load flow [27]. Each generator is controlled by the widely used IEEE type 1 static excitation system. A gas turbine and speed governor control the speed and input torque of each machine. The synchronous machine, excitation system and gas turbine parameters have been obtained from [28], while the governor speed control parameters are tuned against real recorded events as will be explained in the following sub-section. The transmission lines are modeled using $\pi$-sections with lumped resistance, capacitance, and inductance parameters calculated from the power flow data provided by the Electricity Ten Year Statement for 2017 [27] as per the methodology presented in [29]. The model parameters can be found in [30], and hence have not been repeated in this paper.

4.1.2 Validation: The model has been validated by means of two tests [30]:

1. Load flow analysis: This test is to evaluate the steady-state performance of the model. The load flow simulation data of the test system closely matches the winter peak 2017 data [27]. This includes the generation and demand data at each region and the power flow across the boundaries of the regions.

2. Dynamic frequency response evaluation: The dynamic response of the reference power system model is benchmarked against a multiple real historic frequency deviation events, with data recorded by phasor measurement units located at various points of the GB power transmission network. In [30], the event chosen was the trip of the England-France high voltage direct current inter-connector on 11th of January 2016 leading to a power loss of 900 MW. In other words, the model represents the real-world GB network on the 11th of January, 2016. The total generation and demand of the model is adjusted to match the values on the day of the event (total demand $=59.56 \mathrm{GW}$ ). The inertia constant, the governor time constant, the droop percentage, and the load reference set-point parameters are tuned to ensure the model frequency response matches the frequency response (pre-disturbance RoCoF and frequency nadir) obtained from the phasor measurement units.

\subsection{Control Implementation}

The following controls are implemented in each of the five areas of the GB power system with nominal frequency $f_{\text {nom }}=50 \mathrm{~Hz}$. The parameters utilized are presented below.

1. Primary Control Loop: The synchronous generators in each of the areas participate proportionally to their capacity to contain the frequency with a droop setting of $13 \%$.

2. Secondary Control Loop: The typical values of $K_{P i}$ and $T_{I i}$ are in the range of $0.1-1.0$ and $50-200$ s respectively [31], [32]. The best (fastest) value of $T_{I}=50 \mathrm{~s}$ has been chosen. The value of $K_{P i}$ has been chosen as 0.1 . Both the synchronous generator and aggregated load constitute the secondary reserves with $\alpha_{C G i}=0.25$ and
3. Balance Control Loop: With aggregated loads participating as reserves, the values of $K_{P, a u x}$ and $T_{I, a u x}$ are selected as 0.1 and 5 s respectively. With no ramp-rate constraints, a smaller value of $T_{I, \text { aux }}$ has been chosen to demonstrate the effectiveness of BCL. For the ADD, the values of $\Delta P_{T h, i}^{t i e}=50 \mathrm{MW}, T_{n}=0.02 \mathrm{~s}$ and $T_{f}=0.02 \mathrm{~s}$ are selected.

4. Delays: Measurement and communication delays are incorporated within the secondary and balance control loop. The values of $\mathrm{ACE}$ and $\Delta P^{t i e}$ are thus updated every $2 \mathrm{~s}$ representing the measurement delay [34], while the outputs of the two control loops, $\Delta P_{c i}$ and $\Delta P_{i}^{D S A}$, are delayed by $1 \mathrm{~s}$ representing communications delay [35].

\subsection{Incorporation of Renewable Generation Penetration}

To analyze the performance and aptness of the proposed frequency control approach in a more representative power system, the incorporation of renewable generation within the test power system is necessary. In this sub-section, the incorporation of the impact of renewable generation penetration, in particular the reduction in inertia, within the test power system is explained. First, a multiplier $\mu_{\text {inertia }}$ is defined that scales the system inertia constant $(H)$ such that $H=\mu_{\text {inertia }} H_{0}$, where $H_{0}$ is the reference system inertia. To elaborate, for example, a value of $\mu_{\text {inertia }}=0.9$ leads to a $10 \%$ reduction in system inertia. Accordingly, the inertia constant and power generation of each of the six-machines within the test power system is reduced by $10 \%$. As sub-cycle phenomena are not under consideration, a simpler first-order equivalent model representing non-synchronous generation [36] in each of the areas provide the remainder of the power generation.

\subsection{Demand Side Aggregators (DSAs)}

DSAs in this work correspond to energy management systems for active residential customers and/or commercial buildings (referred to as prosumers) with fast-acting responsive loads modeled as dynamic loads in simulation. The response dynamics of DSAs, i.e., the response delay and uncertainties, are subject to accurate load modeling and capacity estimation, that are by nature time-varying and an important field of research on its own. Two types of delays contribute towards the response delay of DSAs; the communication delays between the DSA and the participating prosumer load, and the response time (on/off delay) of the prosumer load upon receipt of activation command from DSA. The response characteristics of a prosumer load, such as the minimum time a load has to stay on/off after activation or the minimum time before the same load can be called on for a second activation, are considered a part of DSA capacity estimation that is assumed to be guaranteed in principle, i.e., either by regulation or penalty mechanisms enforced by DSA itself. However, the communications delay is network-dependent and should be considered for establishing the performance of frequency control mechanisms. Therefore, in this paper, it is assumed that the required amount of restoration reserves are procured by each of the LFC areas while the participating loads are assumed to respond to the control command immediately with no turn on/off delays [37] considered for this work.

\subsection{Other Characteristics}

A number of occurrences of $\sim 1 \mathrm{GW}$ generation loss have been experienced by the GB grid within the last year and therefore a disturbance magnitude of $1 \mathrm{GW}$ within area 2 has been selected as the reference frequency disturbance [38]. The net generation loss is emulated by a step increase in load and a net load loss by a step decrease. 


\section{Performance Evaluation}

In this section, the applicability of the proposed ELFC is evaluated by means of real-time simulations and experiments within a state-ofthe-art smart grid laboratory. A three step approach for validation is adopted as follows:

1. Simulation and analysis: The feasibility of the approach is verified by means of real-time simulations. The response of the system subject to reference disturbance is evaluated followed by a sensitivity analysis with respect to system inertia and size of disturbance. This is followed by bringing forth the advantages offered by the key features of the BCL, i.e., the ADD and use of tie-line power flow $\left(\Delta P_{i}^{t i e}\right)$ as control input instead of the ACE.

2. Controller Hardware-in-the-Loop validation: Such a validation serves two main purposes: (i) the validation of the controller under realistic delays and (ii) the prototyping of the proposed control within an actual hardware controller to demonstrate its real-world application.

3. Controller and Power Hardware-in-the-Loop validation: The performance improvement of the proposed ELFC over CLFC has been achieved by means of incorporation of BCL that relies on ADD for a faster disturbance detection. The disturbance detection utilizes RoCoF, that is regarded as a very noisy measurement. Any control that relies on RoCoF should therefore be validated by means of real measurements enabled by controller and power hardware-in-theloop implementation. Therefore, the ADD of the proposed control is validated in this way.

In all the above cases, the performance of the proposed ELFC is compared to that of CLFC. To aid the assessment, two key indicators are defined, namely (i) frequency restoration time $\left(T^{\text {rest }}\right)$ and (ii) relative frequency overshoot $\left(\Delta f^{\text {ovr }}\right)$. To asses the $T^{\text {rest }}$ of the controllers, an error margin $\epsilon=0.01$ is defined such that $T^{\text {rest }}$ is the time interval between the initiation of the disturbance to the point when $|\Delta f|=\left|f_{\text {meas }}-f_{\text {nom }}\right|<\epsilon$, and can be represented as

$$
T^{r e s t}=\left\{T^{r e s t} \in \mathcal{R}: \forall t>T^{r e s t},|\Delta f| \leq \epsilon\right\}
$$

Defining the minimum and maximum frequency after a disturbance as $f_{\max }$ and $f_{\min }$ respectively, the relative overshoot is calculated as

$$
\Delta f^{\text {ovr }}=\left|\frac{f_{\max }-f_{\text {nom }}}{f_{\text {nom }}-f_{\min }}\right|
$$

As the GB power system is a relatively strongly coupled network, i.e. a stiff network; therefore the same frequency characteristics are observed throughout the network [39]. For simplicity and clarity of the figures, only the frequency of the area with a disturbance is plotted for the analysis that follows.

\subsection{Simulation and analysis}

5.1.1 Response to reference disturbance: The system frequency responses subject to a generation loss at $t=10 \mathrm{~s}$ and a load loss at $t=110$ s for CLFC and ELFC are presented in Fig. 4a. The restoration time for the CLFC is $83 \mathrm{~s}$ with no overshoot. The proposed ELFC, by means of fast and accurate detection of disturbance location, contributes to improving frequency nadir (frequency zenith for load loss) and leads to a faster restoration time. With a restoration time of $35.2 \mathrm{~s}$, the proposed ELFC is twice as fast but presents an overshoot of $35 \%$. An acceptable frequency response of a system subject to a disturbance is defined by an exponentially decaying function $H(s)=f_{n o m} \pm A e^{-\frac{1}{T} t}$ referred to as the trumpet curve, set as a requirement by the European Network of Transmission System Operators for Electricity [31]. Considering the response of the conventional control as reference, trumpet curve parameters are calculated as $A=0.31 \mathrm{~Hz}$ and $T=11 \mathrm{~s}$. As can be observed from Fig. $4 \mathrm{a}$, the response of the proposed ELFC, with a 35\% overshoot, is well within the bound set by the trumpet curve. The overshoot of the ELFC can be abated and the response further improved by means

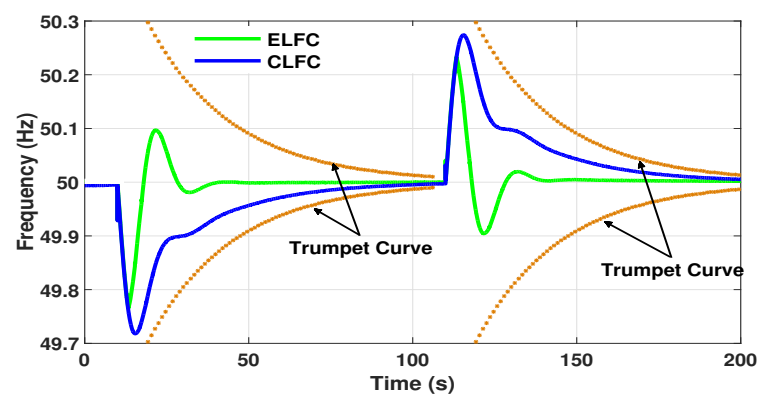

(a) Frequency response.
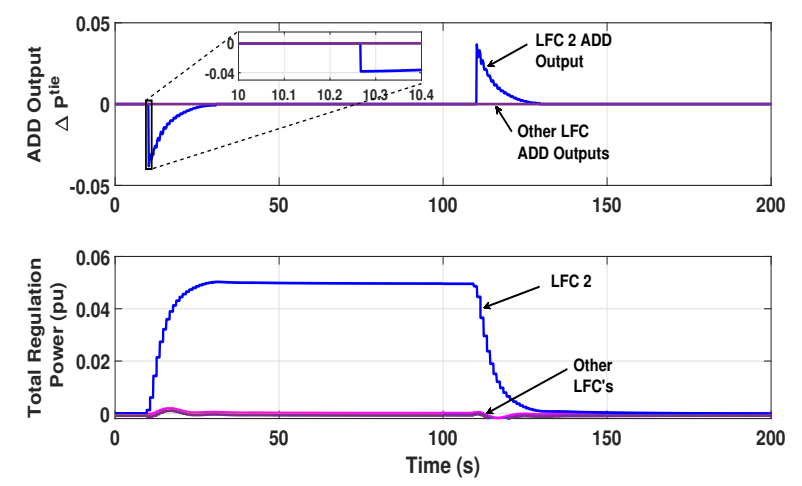

(b) ADD output and total regulation power for ELFC.

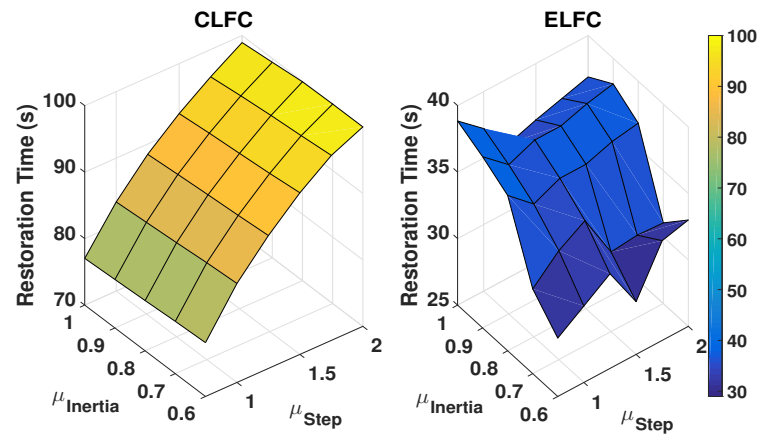

(c) Sensitivity analysis.

Fig. 4: Simulation and sensitivity analysis results for performance evaluation of proposed ELFC.

of faster acting PFC, where primary response is provided by battery energy storage systems as opposed to CGs as is the case for simulations undertaken.

The output of ADD and the total regulation power, i.e., the sum of the regulation command from the DSAs and the secondary control loop, for all the areas subject to the generation loss and load loss is shown in Fig. 4b. As can be observed, only the output of LFC 2 ADD is non-zero (and equal to $\Delta P_{2}^{t i e}$ ) consequent to successful disturbance identification. Furthermore, this identification is within $300 \mathrm{~ms}(\sim 250 \mathrm{~ms})$ as shown. This enables the fast activation of resources through the BCL to be activated unilaterally, i.e., only in area with disturbance, as is evident from the total regulation power shown.

5.1.2 Sensitivity analysis: To further evaluate the performance of the proposed ELFC, a sensitivity analysis for variation in size of disturbance and system inertia is undertaken. The size of disturbance $\left(P^{s t e p}\right)$ is varied by means of a multiplier $\mu_{\text {step }}$ such that $P^{s t e p}=$ $\mu_{\text {step }} P_{0}^{\text {step }}$, where $P_{0}^{\text {step }}$ is the reference disturbance magnitude. In a similar manner, a multiplier $\mu_{\text {inertia }}$, scales the system inertia constant $(H)$ such that $H=\mu_{\text {inertia }} H_{0}$, where $H_{0}=4.68 \mathrm{~s}$. The results of the analysis for CLFC and ELFC are presented in Fig. 4c. As can be observed, the restoration time for CLFC increases with 


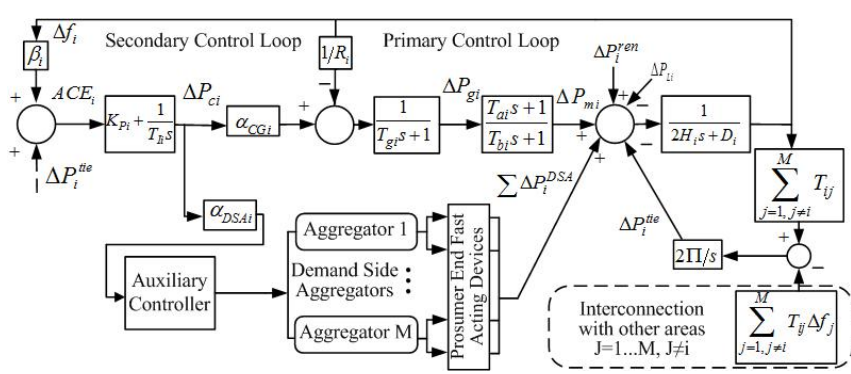

(a) CLFC with auxiliary controller

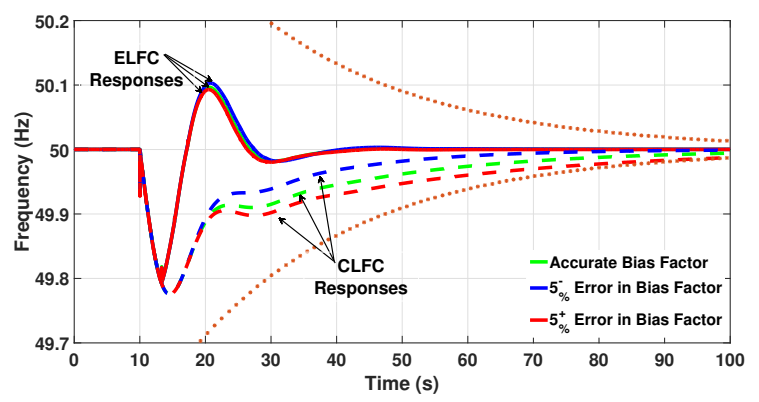

(c) Frequency response

Fig. 5: Results for performance evaluation of proposed ELFC

increasing disturbance size but the decreasing system inertia does not have a significant impact. On the other hand, the performance of proposed ELFC is significantly different, the restoration time of which is not affected by the increase in disturbance size while at the same time, a decrease in system inertia leads to faster restoration times. Therefore, the proposed ELFC is more robust to varying disturbance sizes and system characteristics, a necessity for future power systems.

With variations in disturbance size and system inertia, the relative overshoot for the proposed ELFC is always under $40 \%$ and within the bounds set by the respective trumpet curve. While the CLFC exhibits no overshooting characteristic, with decreasing system inertia, the frequency nadir significantly decreases. The proposed ELFC improves the frequency nadir for all the cases assessed.

5.1.3 Importance of ADD: The objective of this sub-section is to demonstrate that the improvement in the performance of the proposed control is not only due to the incorporation of additional faster auxiliary controller but the fact that the identification of the location of the disturbance does play a very important role. Therefore, the importance of ADD is demonstrated by means of conducting smallsignal stability analysis on the test power system. The two cases for comparison are: (i) the proposed ELFC control and (ii) the CLFC incorporating the auxiliary controller only as shown in Fig. 5a. The state-space model of M-area interconnected power system with BCL (as shown in Fig. 2) can be represented as [40]

$$
\left\{\begin{array}{l}
\dot{x}=A x+B u+F w \\
y=C x
\end{array}\right.
$$

with

$$
\left\{\begin{array}{l}
x=\left[x_{1}, x_{2}, x_{3}, x_{4}, x_{5}\right]^{T} \\
u=\left[u_{1}, u_{2}\right]^{T} \\
w=\left[\Delta P_{L_{1}}, \ldots, \Delta P_{L_{M}}\right]^{T}
\end{array}\right.
$$

where $x$ is the state vector, $u$ is the control vector and $w$ is the disturbance vector. The internal states can be represented as $x_{1}=\left[\Delta f_{1}, \ldots, \Delta f_{M}\right], x_{2}=\left[\Delta P_{m_{1}}, \ldots, \Delta P_{m_{M}}\right]$, $x_{3}=\left[\Delta P_{1}^{t i e}, \ldots, \Delta P_{M}^{t i e}\right], x_{4}=\left[\int A C E_{1}, \ldots, \int A C E_{M}\right]$ and
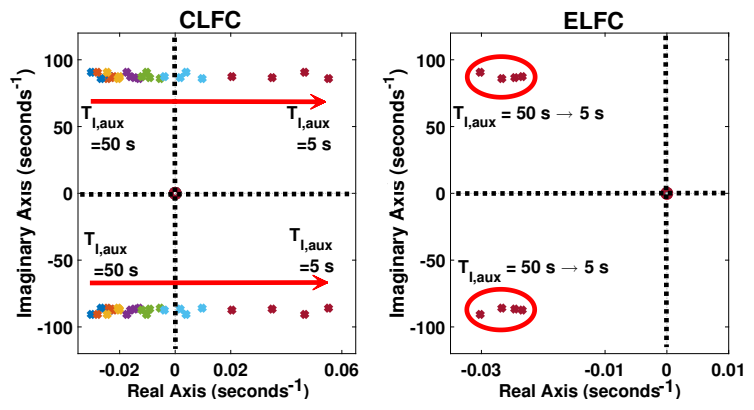

(b) Eigenvalue analysis

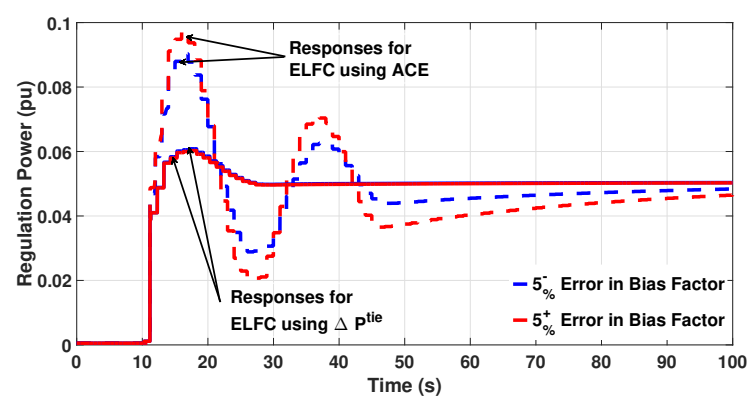

(d) Regulation power

$x_{5}=\left[\int d_{1}, \ldots, \int d_{M}\right]$. The internal inputs can be represented as $u_{1}=\left[\Delta P_{c_{1}}, \ldots, \Delta P_{c_{M}}\right]$ and $u_{2}=\left[\Delta P_{1}^{D S A}, \ldots, \Delta P_{M}^{D S A}\right]$. The coefficient matrices $\mathrm{A}, \mathrm{B}$, and $\mathrm{C}$ can be found in [40]. The system is linearized around $f_{n o m}$ in steady-state. The input to the system is the reference disturbance in area 2 and the output is the system frequency.

The Eigenvalues for the two cases are obtained by varying the $T_{I, \text { aux }}$ from $50 \mathrm{~s}$ to $20 \mathrm{~s}$ in steps of $10 \mathrm{~s}$ and then to $5 \mathrm{~s}$ in steps of $5 \mathrm{~s}$. In Fig. 5b, the pole-zero map for the CLFC (left) and the proposed ELFC (right) have been presented. Only the states that are impacted by $T_{I, a u x}$ are shown for clearer representation. As can be observed from Fig. 5b, for CLFC, as the value of $T_{I, \text { aux }}$ is reduced, the poles move towards the imaginary axis. For $T_{I, \text { aux }}=10 \mathrm{~s}$, the poles cross the imaginary axis representing an unstable system. However, for the proposed ELFC, where the ADD ensures unilateral reserve activations only within the area where the disturbance has occurred, the poles are very slightly impacted by the reduction in $T_{I, \text { aux }}$, which therefore does not deteriorate the stability of the system.

5.1.4 Choosing $\Delta P^{\text {tie }}$ over ACE: This sub-section presents a justification for the use of $\Delta P^{t i e}$ within BCL as opposed to the conventionally used $\mathrm{ACE}$. Consider the frequency responses presented in Fig. 5c, with frequency bias factor miscalculation of $5 \%$ for both the CLFC and proposed ELFC. As can be observed, a 5\% miscalculation of $\beta$ results in a $\pm 17 \mathrm{~s}$ variation in $T^{\text {set }}$ for the CLFC while has no significant impact within the proposed ELFC. $\beta$ is a combination of the droop set for the area and the damping offered by the load within the area; and it is not uncommon for errors in its calculation. Larger generating units within the network are bound by the grid code to provide a set percentage response to a variation in frequency. However, if the units do not abide by the set droop, this will lead to miscalculation of $\beta$. Furthermore, presently it is normal practice to determine $\beta$ on a yearly basis [11]. In future systems, it is expected that the characteristics of the network (e.g. system inertia) will vary vastly even within a single day. It is also expected that the difference between the peak load and the base load will increase significantly [7], thereby resulting in the load offering a different damping effect at different times. Therefore, the proposed ELFC utilizing $\Delta P^{t i e}$ is more resilient to changes expected within the future power system. 


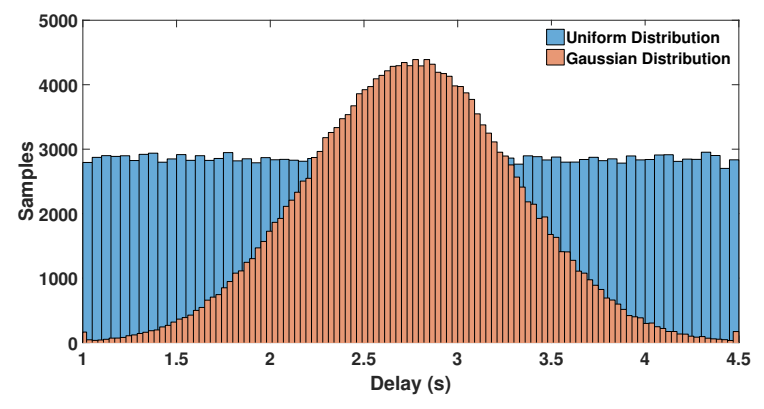

(a) Delay distributions under consideration.

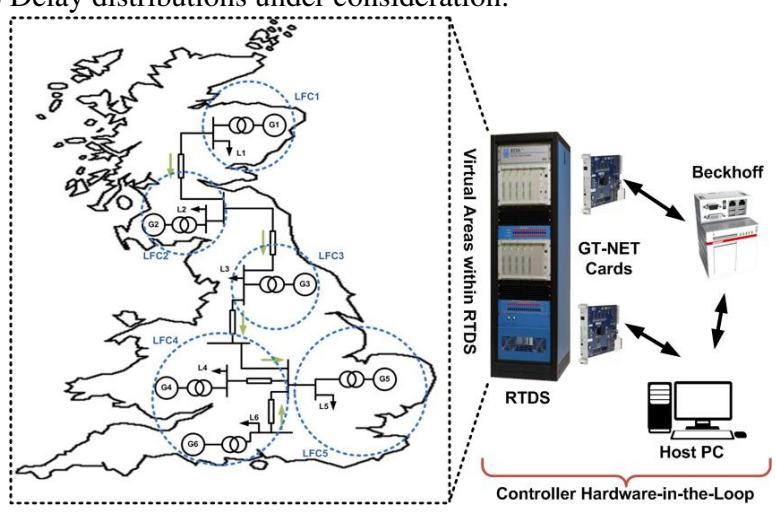

(b) Controller hardware-in-the-loop setup.

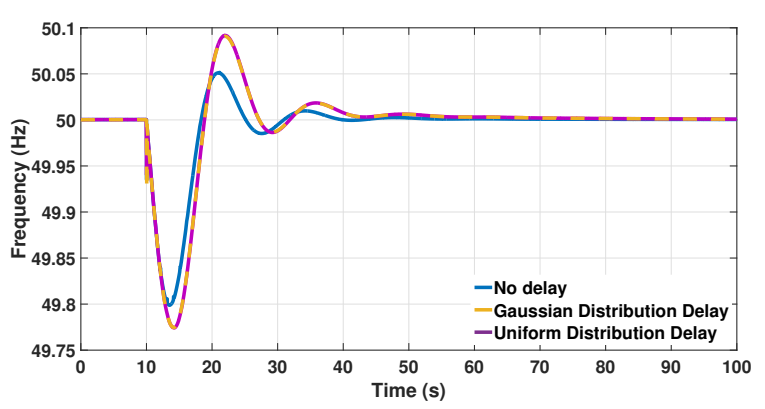

(c) Frequency response with and without delays.

Fig. 6: Setup and results for controller hardware-in-the-loop implementation.

Furthermore, if ACE was instead utilized within the BCL of the proposed scheme, although the activations of reserves are guaranteed to be unilateral within the area where the disturbance has been detected, the miscalculation of bias factor would lead to nuisance activations as shown in Fig. 5d. It should be noted that utilizing $\Delta P^{t i e}$ avoids any nuisance activations.

\subsection{Controller Hardware-in-the-Loop Validation}

A detailed study on experimental validation of ancillary service provision capability of DSAs controlling a portfolio of highly distributed demand side devices has been undertaken in [41]. The study identifies that validation of any control relying on DSAs for provision of time critical ancillary services, should incorporate realistic communication delays to demonstrate robustness. The results presented in previous sub-sections assume a fixed communications delay; however, the communication delay referred to in this paper is the time it takes for a control command from the aggregator to reach the participating device and is highly dependent upon the communications architecture employed by the potential demand side aggregator.

The performance of the communications networks that connect a control centre to the end devices for demand side applications has been analyzed in [42] and the results show that the latency expected
Table 1 Test Metrics

\begin{tabular}{lcc}
\hline \hline Delay & $T^{\text {Rest }}(\mathrm{s})$ & $\Delta f^{\text {ovr }}(\%)$ \\
\hline \hline No delay & 24.16 & 28.7 \\
Gaussian distribution delay & 42.37 & 44 \\
Uniform distribution delay & 42.3 & 43.6 \\
\hline \hline
\end{tabular}

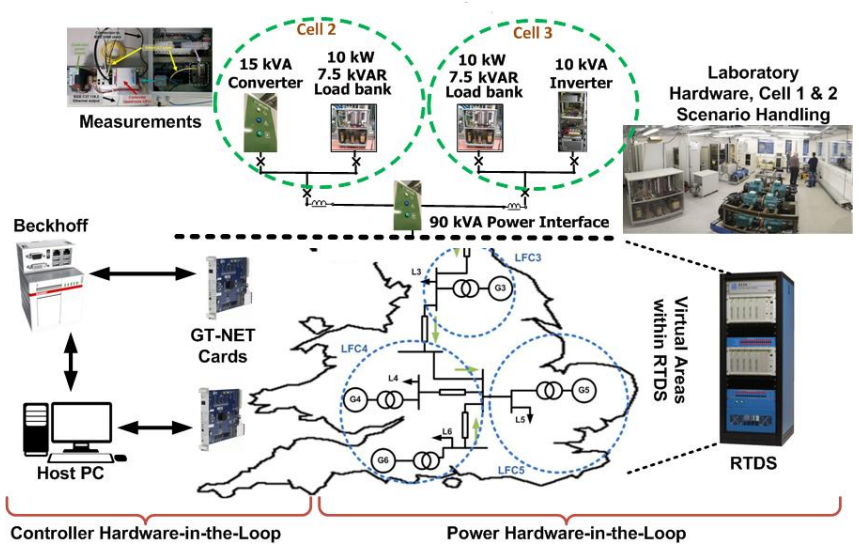

(a) controller and power hardware-in-the-loop setup.

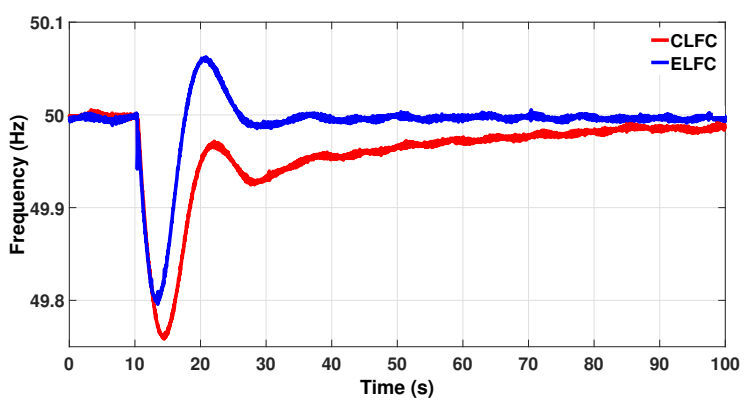

(b) Frequency response.

Fig. 7: Setup and results for controller and power hardware-in-theloop implementation.

is between $1-4.5 \mathrm{~s}$. The analysis presents an estimation of delay but does not provide a mean value for the delay nor a standard deviation. The variation in delay is mostly associated to the number of hops a message packet makes in the neighborhood area network before it reaches the device. As a constant delay is highly unlikely in a real-world scenario, for this work, two types of distributions are considered as shown in Fig. 6a, a uniform distribution and a Gaussian distribution where the mean value $\mu$ has been chosen as 2.75 s (average of minimum and maximum latency) with a standard deviation of $\sigma$ of $0.3 \mathrm{~s}$.

A controller hardware-in-the-loop implementation, as presented in Fig. 6b, is utilized. The five-areas of the GB power system are simulated within the digital real-time simulator, the proposed ELFC is implemented within a Beckhoff real-time controller while a simple transactive energy based DSA runs on the host PC. The proposed ELFC is run at a time step of $10 \mathrm{~ms}$ with $T_{I, \text { aux }}=5 \mathrm{~s}$ while all other control parameters remain the same as in the simulation study. The response of the system subject to the reference imbalance is presented in Fig. 6c and test metrics in Table 1. As can be observed, the response of the proposed ELFC incorporating delays characterized for demand side applications is stable and satisfactory, and there is not much difference in response with two different delay distributions. The deployment of the control on dedicated controller hardware enabled by rapid prototyping, further demonstrates the viability of the control for real-world implementations. 


\subsection{Controller and Power Hardware-in-the-Loop}

The controller and power hardware-in-the-loop setup is shown in Fig. 7a. As can be observed, two areas of the GB network are represented by Cell 2 and Cell 3 of the Dynamic Power Systems Laboratory [43] while the remaining three areas are simulated within the digital real-time simulator. To ensure stability of the setup and to be able to represent a large portion of the GB network, the currents are scaled by a factor of $k=0.8 \times 10^{6}$. A detailed description and process of initialization and synchronization of the power hardware-in-the-loop setup is found in [44].

The system is subject to disturbance in LFC area 2 (Cell 2 in hardware, Fig. 7a), a loss of generation, with magnitude of 800MW (1000W in hardware). A smaller magnitude of disturbance in experimental evaluation compared to the reference imbalance magnitude is due to the stability of the setup, which put a limit on the spare power capacity reserved for imbalance event. As the objective of power hardware-in-the-loop evaluation is to demonstrate the capability of the proposed control to operate under real measurements, the magnitude of the disturbance does not impact the integrity of the study. However, it further demonstrates the applicability of the proposed solution to a wide range of disturbances. The frequency measurements are derived from the raw voltage measurements at Cell 2 and Cell 3 nodes using 3-phase voltage transformers. A realtime system controller, a multi-processor based system, with analog to digital sampling and digital pre-filtering at $666.66 \mu \mathrm{s}$ is utilized to process the measurements. As in the simulation based studies, the measurement window to obtain RoCoF remains $T_{n}=0.02 \mathrm{~s}$. The parameters of the control system remain same as in the previous study. The system frequency response subject to generation loss at $t=10$ s is shown in Fig. 7b. As can be observed, the ADD was able to identify the imbalance evident from the restoration of frequency much faster than CLFC. Therefore, demonstration of the feasibility of ADD operation with real measurements strongly supports the deployment of the proposed control approach in future power systems.

\section{Conclusions}

In this paper, to improve the speed of response of secondary frequency control, a novel control has been proposed. The control incorporates a fast acting balance control loop, in addition to the secondary control loop. The balance control loop incorporates an area distrubance determiner capable of fast, autonomous and definitive identification of location of the imbalance. The impact of fast and definitive identification of location of imbalance is two-fold:

- it ensures unilateral activation of reserves only within the area where the imbalance initiated. This allows for faster response speed. - it enables use of area power imbalance observed over tie-lines as control input as opposed to conventionally utilized area control error. This avoids sympathetic activations even at faster response speeds.

The proposed enhanced load frequency control further decouples the control effort of fast acting resources from that of conventional generators. The control effort to fast acting resources is a combination of effort from balance control loop and secondary control loop, while the control effort to conventional generators is from secondary control loop only. This allows for the exploitation of true effectiveness of fast acting devices for frequency restoration. The analytical assessments in the work undertaken provides a high degree of confidence in the aptness of the proposed approach for dynamically changing power systems while the experimental evaluation demonstrates its real-world applicability.

\section{Acknowledgment}

This work was supported by the European Commission under the FP7 ELECTRA IRP (grant no: 609687). Any opinions, findings and conclusions or recommendations expressed in this material are those of the authors and do not necessarily reflect those of the European Commission.

\section{References}

1 'National Transmission Network Development Plan,'. (Australian Energy Market Operator (AEMO), 2016). Available from: http://www.aemo.com. au/Electricity/National-Electricity-Market-NEM/Planning-and-forecasting/ National-Transmission-Network-Development-Plan

2 Torres, L.M.A., Lopes, L.A.C., MorÃąn, T.L.A., Espinoza, C.J.R.: 'Self-tuning virtual synchronous machine: A control strategy for energy storage systems to support dynamic frequency control', IEEE Transactions on Energy Conversion, 2014 29, (4), pp. 833-840

3 Rakhshani, E., Remon, D., Cantarellas, A.M., Rodriguez, P.: 'Analysis of derivative control based virtual inertia in multi-area high-voltage direct current interconnected power systems', IET Generation, Transmission Distribution, 2016, 10, (6), pp. $1458-1469$

4 Alipoor, J., Miura, Y., Ise, T.: 'Power system stabilization using virtual synchronous generator with alternating moment of inertia', IEEE Journal of Emerging and Selected Topics in Power Electronics, 2015, 3, (2), pp. 451-458

5 Soni, N., Doolla, S., Chandorkar, M.C.: 'Improvement of transient response in microgrids using virtual inertia', IEEE Transactions on Power Delivery, 2013, 28 , (3), pp. 1830-1838

6 'Fast Frequency Response in the NEM Working Paper,'. (Australian Energy Market Operator (AEMO), 2017). Available from: https://www.aemo.com.au/-/media/Files/Electricity/NEM/SecurityandReliability/ Reports/2017/FFR-Working-Paper---Final.pdf

7 'System Operability Framework 2016,' (National Grid, 2016). Available from: http://www2.nationalgrid.com/UK/Industry-information/Future-of-Energy/ System-Operability-Framework/

8 ' $\mathrm{RoCoF}$ - An independent analysis on the ability of Generators to ride through Rate of Change of Frequency values up to 2Hz/s,'. (DNV-KEMA for EirGrid/SONI, 2013). Available from: http://www.eirgridgroup.com/site-files/library/ EirGrid/DNV-KEMA_Report_RoCoF_20130208final_.pdf

9 'International Review of Frequency Control Adaptation,'. (DGA Consulting report to Australian Energy Market Operator (AEMO), 2016) Available from: https://www.aemo.com.au/-/media/Files/Electricity/NEM/SecurityandReliability/ Reports/FPSS---International-Review-of-FrequencyControl.pdf

10 'Policy P1: Load-Frequency Control and Performance'. (in Continental Europe Operation Handbook, ENTSO-E, 2016). Available from: https://www.entsoe.eu/fileadmin/user_upload/_library/publications/entsoe/ Operation_Handbook/Policy_1_final.pdf

11 'Balancing and Frequency Control,'. (North American Electric Reliability Corporation, 2011). Available from: http://www.nerc.com/docs/oc/rs/NERC\% 20Balancing\%20and\%20Frequency\%20Control\%20040520111.pdf

12 'Guide to Ancillary Services in the National Electricity Market,. (Australian Energy Market Operator (AEMO), 2015). Available from: https://www.aemo.com.au/-/media/Files/PDF/ Guide-to-Ancillary-Services-in-the-National-Electricity-Market.ashx

13 Jin, C., Lu, N., Lu, S., Makarov, Y.V., Dougal, R.A. 'A coordinating algorithm for dispatching regulation services between slow and fast power regulating resources', IEEE Transactions on Smart Grid, 2014, 5, (2), pp. 1043-1050

14 Guo, Y., Xie, X., Wang, B., Shi, W., Dong, Y., Mou, L., et al. 'Improving agc performance of a coal-fueled generators with mw-level bess'. In: 2016 IEEE Power Energy Society Innovative Smart Grid Technologies Conference (ISGT). (, 2016. pp. $1-5$

15 Xie, X., Guo, Y., Wang, B., Dong, Y., Mou, L., Xue, F.: 'Improving agc performance of coal-fueled thermal generators using multi-mw scale bess: A practical application', IEEE Transactions on Smart Grid, 2018, 9, (3), pp. 1769-1777

16 Zhang, F., Hu, Z., Xie, X., Zhang, J., Song, Y.: 'Assessment of the effectiveness of energy storage resources in the frequency regulation of a single-area power system', IEEE Transactions on Power Systems, 2017, 32, (5), pp. 3373-3380

17 Kintner.Meyer, M.: 'Regulatory policy and markets for energy storage in north america', Proceedings of the IEEE, 2014, 102, (7), pp. 1065-1072

18 'Frequency Control by Demand Management (FCDM),'. (National Grid, 2015). Available from: http://www2.nationalgrid.com/WorkArea/DownloadAsset.aspx? $\mathrm{id}=41294$

19 Short, J.A., Infield, D.G., Freris, L.L.: 'Stabilization of grid frequency through dynamic demand control', IEEE Transactions on Power Systems, 2007, 22, (3), pp. 1284-1293

20 Hu, J., Cao, J., Guerrero, J.M., Yong, T., Yu, J.: 'Improving frequency stability based on distributed control of multiple load aggregators', IEEE Transactions on Smart Grid, 2017, 8, (4), pp. 1553-1567

21 Variani, M.H., Tomsovic, K.: 'Distributed automatic generation control using flatness-based approach for high penetration of wind generation', IEEE Transactions on Power Systems, 2013, 28, (3), pp. 3002-3009

22 Xie, P., Li, Y., Zhu, L., Shi, D., Duan, X.: 'Supplementary automatic generation control using controllable energy storage in electric vehicle battery swapping stations', IET Generation, Transmission Distribution, 2016, 10, (4), pp. 1107-1116

23 Keyhani, A., Chatterjee, A.: 'Automatic generation control structure for smart power grids', IEEE Transactions on Smart Grid, 2012, 3, (3), pp. 1310-1316

24 Kok, K., Widergren, S.: 'A society of devices: Integrating intelligent distributed resources with transactive energy', IEEE Power and Energy Magazine, 2016, 14, (3), pp. 34-45

25 R. L..Graham, Knuth, D.E., Patashnik, O.: 'Concrete Mathematics: A Foundation for Computer Science'. (Addison-Wesley Publishing Company, 1990). Available from: https://www.csie.ntu.edu.tw/ r97002/temp/Concrete\% 20Mathematics\%202e.pdf 
26 Prostejovsky, A.M., Marinelli, M., Rezkalla, M., Syed, M.H., Guillo.Sansano, E.: 'Tuningless load frequency control through active engagement of distributed resources', IEEE Transactions on Power Systems, 2018, 33, (3), pp. 2929-2939

27 'Electricity Ten Year Statement 2016",'. (National Grid, 2016). Available from: http://www2.nationalgrid.com/UK/Industry-information/Future-of-Energy/ Electricity-ten-year-statement

28 Kundur, P.: 'Power System Stability and Control'. (McGraw-Hill Education, 1994). Available from: https://books.google.co.uk/books?id=2cbvyf8Ly4AC

29 Xia, J., Dysko, A. 'Uk transmission system modelling and validation for dynamic studies'. In: IEEE PES ISGT Europe 2013. (, 2013. pp. 1-5

30 Emhemed, A., Adam, G., Hong, Q., Burt, G. 'Studies of dynamic interactions in hybrid ac-dc grid under different fault conditions using real time digital simulation'. In: 13th IET International Conference on AC and DC Power Transmission (ACDC 2017). (, 2017. pp. 1-5

31 'UCTE Operation Handbook Policy 1: Load-frequency control and performance,'. (European Network of Transmission System Operators for Electricity, 2017). Available from: https://www.entsoe.eu/publications/system-operations-reports/ \#continental-europe-operation-handbook

32 'UCTE Operation Handbook Appendix 1: Load-Frequency Control and Performance,'. (European Network of Transmission System Operators for Electricity, 2017). Available from: https://www.entsoe.eu/publications/ system-operations-reports/\#continental-europe-operation-handbook

33 'The Power of Transformation Wind, Sun and the Economics of Flexible Power Systems,'. (International Energy Agency, 2014). Available from: https://www.iea. org/publications/freepublications/publication/The_power_of_Transformation.pdf

34 Zhang, C.K., Jiang, L., Wu, Q.H., He, Y., Wu, M.: 'Delay-dependent robust load frequency control for time delay power systems', IEEE Transactions on Power Systems, 2013, 28, (3), pp. 2192-2201

35 Ko, K.S., Sung, D.K.: 'The effect of cellular network-based communication delays in an ev aggregator's domain on frequency regulation service', IEEE Transactions on Smart Grid, 2017, pp. 1-1

36 M. J..E..Alam, Muttaqi, K.M., Sutanto, D.: 'A novel approach for ramp-rate control of solar pv using energy storage to mitigate output fluctuations caused by cloud passing', IEEE Transactions on Energy Conversion, 2014, 29, (2), pp. 507-518

37 Lu, N., Zhang, Y.: 'Design considerations of a centralized load controller using thermostatically controlled appliances for continuous regulation reserves', IEEE Transactions on Smart Grid, 2013, 4, (2), pp. 914-921

38 'National Electricity Transmission System Performance Report 2015-2016 âĂS Report to the Gas and Electricity Markets Authority,'. (National Grid, 2016). Available from: http://www2.nationalgrid.com/WorkArea/DownloadAsset.aspx? id $=8589936830$

39 P. M.Ashton, Saunders, C.S., Taylor, G.A., Carter, A.M., Bradley, M.E.: 'Inertia estimation of the gb power system using synchrophasor measurements', IEEE Transactions on Power Systems, 2015, 30, (2), pp. 701-709

40 Ramana, N.V.: 'Power System Operation and Control'. (Pearson Education India, 2010). Available from: https://books.google.co.uk/books/about/Power_System_ Operation_Control.html?id=Gox3x1ClV94C\&redir_esc=y

41 Syed, M.H., Burt, G.M., D'hulst, R., Verbeeck, J. 'Experimental validation of flexibility provision by highly distributed demand portfolio'. In: CIRED Workshop 2016. (, 2016. pp. 1-4

42 Dambrauskas, P., Syed, M.H., Blair, S.M., Irvine, J.M., Abdulhadi, I.F., Burt, G.M., et al.: 'Impact of realistic communications for fast-acting demand side management', CIRED - Open Access Proceedings Journal, 2017, 2017, (1), management',

43 'Dynamic Power Systems Laboratory,'. (University of Strathclyde, Institute for Energy and Environment, 2018). Available from: https://www.ulabequipment. $\mathrm{com} /$ facility/dynamicpowersystems

44 GuilloSansano, E., Syed, M.H., Roscoe, A.J., Burt, G.M.: 'Initialization and synchronization of power hardware-in-the-loop simulations: A great britain network case study', Energies 2018, 2018, 11, (5), pp. 1087 